\title{
TRANSCRIPTION \\ Relax, it's just a small cut
}

Of 6,545
putative
AR-bound
enhancer sites,
$96 \%$ bound
TOP 1

New research in Cell identifies a role for single-strand nicks mediated by DNA topoisomerase 1 (TOP1) in relaxing supercoiled DNA at gene enhancers to promote enhancerdependent transcription. Subsequent recruitment of the DNA damage response machinery to enhancers ensures that single-strand nicks are repaired quickly.

Research in the past few years has shown that enhancers - distal regulatory regions located upstream and downstream of target genes, to which specific transcription factors

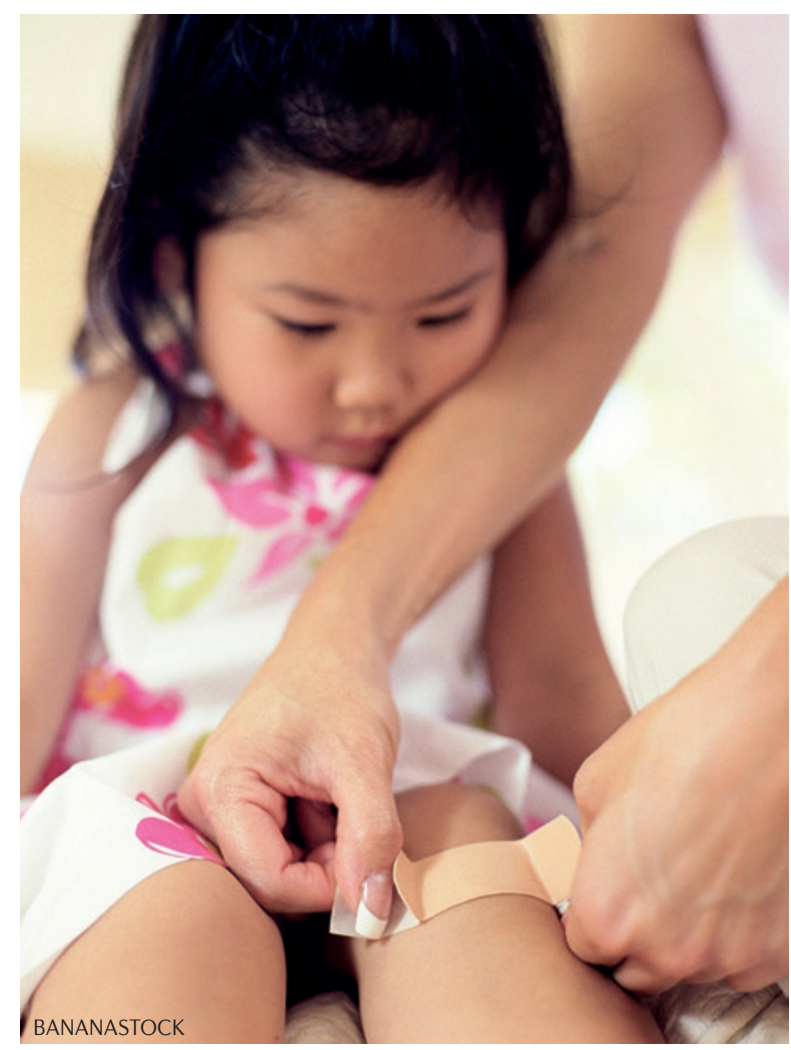

bind - can also function as transcription units for the production of long non-coding RNAs known as enhancer RNAs (eRNAs). In addition to other possible roles, eRNAs seem to stabilize the chromatin loops that juxtapose enhancer and promoter sequences for optimal transcription of the target genes. However, both nucleosome depletion at enhancers and eRNA synthesis can result in DNA supercoiling, which can impede transcription. Rosenfeld and colleagues describe a crucial role for TOP1 in countering this restriction.

In the androgen-dependent prostate adenocarcinoma cell line LNCaP, TOP1 was recruited to androgen receptor (AR)-regulated enhancers in response to androgen treatment. Of 6,545 putative AR-bound enhancer sites, 96\% bound TOP 1 and $60 \%$ showed an androgen-stimulated increase in TOP1 binding as well as in RNA polymerase II occupancy, which is indicative of an active transcriptional unit. In total, 644 enhancers that had significantly upregulated eRNA levels in response to androgen treatment were identified in hormone-starved LNCaP cells. Knockdown of TOP1 decreased eRNA transcription of at least $79 \%$ of these enhancers, accompanied by decreased transcription of 368 target coding genes. The role of TOP1 in promoting the transcription of eRNAs and thus of mRNAs was confirmed for four AR-regulated enhancers of KLK3, KLK2, TMPRSS2 and NDRG1. Prior binding by the androgen-regulated pioneer transcription factor NKX3.1 was required to recruit TOP1 to enhancers following androgen treatment, and NKX3.1 depletion attenuated eRNA and mRNA transcription.

A catalytic dead TOP1 mutant (Y723F) failed to re-instate eRNA induction in TOP1-depleted cells, which suggests that the nicking activity of TOP1 is required for its effects on enhancer activation. Indeed, labelling of DNA breaks and nicks with biotinylated dUTPs was observed at several AR-regulated enhancers after androgen treatment, and was markedly reduced by TOP 1 depletion. Finally, as single-strand nicks could lead to the formation of deleterious double-strand breaks in the DNA, the authors showed that components of the DNA damage response - meiotic recombination 11 (MRE11), RAD50 and ataxia telangiectasia and Rad3-related (ATR), among others - are recruited to AR-regulated enhancers after androgen treatment and are required for eRNA and mRNA transcription.

In summary, the recruitment of TOP 1 and components of the DNA damage response to NKX3.1occupied, AR-regulated enhancers facilitates enhancer activation by creating single-strand DNA nicks that are postulated to relieve transcription-induced torsional stress.

Kirsty Minton

ORIGINAL RESEARCH PAPER Puc, J. et al. Ligand-dependent enhancer activation regulated by topoisomerase-l activity. Cell http://dx.doi. org/10.1016/j.cell.2014.12.023 (2015) FURTHER READING Heinz, S. et al. The selection and function of cell type-specific enhancers. Nature Rev. Mol. Cell Biol. http://dx.doi. org/10.1038/nrm3949 (2015) 